\title{
The influence of prostatic calculi on lower urinary tract symptoms and sexual dysfunction: a narrative review
}

\author{
Hao Wang ${ }^{1,2}$, Ming $M^{1,2}$, Feng Qin $^{1}$, Jiuhong Yuan ${ }^{1,2}$ \\ ${ }^{1}$ Andrology Laboratory, West China Hospital, Sichuan University, Chengdu, China; ${ }^{2}$ Department of Urology, West China Hospital, Sichuan \\ University, Chengdu, China \\ Contributions: (I) Conception and design: J Yuan; (II) Administrative support: J Yuan; (III) Provision of study materials or patients: H Wang, J Yuan; (IV) \\ Collection and assembly of data: H Wang, M Ma; (V) Data analysis and interpretation: H Wang, M Ma, F Qin; (VI) Manuscript writing: All authors; \\ (VII) Final approval of manuscript: All authors. \\ Correspondence to: Jiuhong Yuan. Andrology Laboratory, West China Hospital, Sichuan University, Chengdu, China. Email: jiuhongyuan2107@163.com.
}

\begin{abstract}
Prostatic calculi (PC) are commonly found in patients who present for urologic consultation. However, the effect of PC on urinary symptoms remains controversial. In this study, we searched the Embase and PubMed databases for literature related to the following keywords: "prostatic calculi", "prostatic stone", "prostatic lithiasis" and "prostatic calcification", along with the limits, "lower urinary tract symptoms", "sexual dysfunction", "erectile dysfunction", "erectile function”, and "premature ejaculation”. According to the literature, there are various subtypes of PC based on X-ray or ultrasound findings, including type I/II, type A/B, and endogenous PC/extrinsic PC. Furthermore, the formation of PC remains unclear, and more importantly, the ability of PC to cause lower urinary tract symptoms (LUTS) and sexual dysfunction (SD) is worth exploring. We retrospectively reviewed all available literature and found that most studies agreed that PC are associated with LUTS. The factors which may play an important role in the pathogenesis of LUTS include the size and location of PC, induced inflammation, and the blood flow of the prostate. Similarly, SD was also examined in the patients with PC, and psychological factors cannot be ignored in this regard. However, more in-depth study of the molecular mechanisms, including prospective, controlled, longitudinal, and large- sample studies, are needed in the future.
\end{abstract}

Keywords: Prostatic calculi (PC); lower urinary tract symptoms; sexual dysfunction

Submitted Jul 27, 2020. Accepted for publication Dec 02, 2020.

doi: $10.21037 /$ tau-20-1046

View this article at: http://dx.doi.org/10.21037/tau-20-1046

\section{Introduction}

In the past, prostatic calculi (PC) had received only minor attention. However, with the advent of transrectal ultrasound (TRUS) and computerized tomography (CT), they have garnered greater focus from both patients and urologists. The earliest description of PC was reported by Donatus in 1586 (1). At present, the definition and classification of PC has not been standardized. The terms "prostatic calculi", "prostatic calcification", and "prostatic stones" are variably used to describe hyper-echoic calcium deposits in the prostate (2). In clinical practice, most patients with PC present in health examination centers or urology clinics. PC are usually asymptomatic, but are commonly found in patients with benign prostate hyperplasia, chronic pelvic pain syndrome, prostatitis, or prostate radiotherapy $(3,4)$. The incidence of PC varies widely from $7 \%$ to $70 \%$ across different studies, and about $51.65 \%$ of men among the healthy Han Chinese population suffer from PC $(3,5,6)$. Experts have classified PC into different types and categories. Vilches et al. classified PC into type I (a lobular

$\wedge$ ORCID: 0000-0001-6052-0321. 
surface composed of small spheres) and type II (a larger, multifaceted surface) using an energy dispersive type X-ray micro-analyzer (7). Also, Harada et al. divided PC into type A (discrete small reflection) and type B (large multireflective mass) by TRUS (8). According to crystallography analysis, PC are often also classified into endogenous PC and extrinsic PC. Endogenous PC are primarily formed by prostatic secretions, while extrinsic $\mathrm{PC}$ result from urinary reflux and are often larger than endogenous PC $(3,9,10)$.

Lower urinary tract symptoms (LUTS) and sexual dysfunction (SD) have been brought into focus during PC research. LUTS are a syndrome of storage or voiding problems, and affect approximately $50-70 \%$ of men over the age of 50. Furthermore, the medical expenses brought on by the high prevalence rate of LUTS present a huge burden to both patients and society (11-13). Notably, there are numerous studies reporting on the pathophysiological mechanism of LUTS; however, a unified perspective is still lacking. Coincidentally, SD, a multi-factorial disease that is not only caused by an organic disorder or disease, may also exist in patients with $\mathrm{PC}$, with premature ejaculation and erectile dysfunction (ED) representing the two most common types of SD in men $(14,15)$.

PC might play an important role in the pathogenesis of LUTS and SD. To our knowledge, although numerous articles have provided an overview of the association between PC and LUTS, they have not verified the relationship between LUTS and the inflammatory response, the size and location of PC, or prostate blood flow. Furthermore, few authors have investigated the association between PC and SD. The aim of this paper was thus to review the available literature in order to summarize the relationship between PC and LUTS, and between PC and $\mathrm{SD}$, and to expound the relevant mechanisms. We present the following article in accordance with the NARRATIVE REVIEW reporting checklist (available at http://dx.doi. org/10.21037/tau-20-1046).

\section{Methods}

In order to detect the impact of PC on LUTS and SD, we obtained all relevant articles by comprehensively searching the PubMed and EMBASE databases from 1979 to 2020 using the keywords, "prostatic calculus", "prostatic calculi", "prostatic stone", "prostatic lithiasis", and "prostatic calcification", along with the limits, "lower urinary tract symptoms", "sexual dysfunction", "erectile dysfunction", "erectile function", and "premature ejaculation". We screened 58 articles. The language of the articles was primarily limited to English. We mainly focused on the publications and iconic articles from the past 10 years, and summarized the relevant information from these and other related articles.

\section{Formation of PC}

The results of the reviewed studies suggested that type I PC may be caused by the obstruction of prostatic secretions around benign prostatic hyperplasia or occlusion caused by chronic inflammation (1), and that type II PC may be associated with urinary reflux, which leads to the formation of PC via changing local ion and pondus hydrogenii $(2,9)$. Other studies have analyzed the chemical composition of PC and consistently reported that calcium phosphate stones were the main components of PC (16). However, there does exist an inconsistency, as calcium phosphate is not a component of prostate secretion. Köseoğlu et al. found that PC contained many ingredients that were found only in urine and not in prostate secretions (17). Consequently, they hypothesized that urine obstruction and stasis in the prostate glands contribute to the formation of PC by promoting calcification of the corpora amylacea and the precipitation of crystals (17). Tang et al. reported that age and the anteroposterior diameter of the prostate were stronger risk factors for PC than other factors, with bilirubin level being the only protective factor found in the study after adjustment for other factors (5). Moreover, metabolic disorders could contribute to the formation of PC, and serum uric acid might participate in the formation of PC (18-21). Moreover, Qian et al. speculated that patients with PC often seek medical consultation due to psychological or emotional reasons, and thus experience a decreased frequency of sexual activity with increasing depression, which ultimately leads to the excretion of prostatic secretions that are involved in the formation of PC (22).

\section{PC and LUTS}

In recent decades, LUTS with PC has garnered increasing attention from authors, however, the effect of PC on LUTS remains controversial. Some studies reported that PC were not a predictive factor of severe LUTS, and independent of prostatic inflammation $(23,24)$. Park et al. enrolled 802 patients with LUTS into their study using multivariate 
analysis, and the International Prostatic Symptoms Score (IPSS) exhibited no statistical differences. Meanwhile, the differences in age and prostate volume were significant, and thus they believed that patients with PC might have developed LUTS not only from PC but also because of age and other factors (24). Kim et al. believed that inflammation of the prostate aggravated LUTS, but the degree of inflammation had nothing to do with the type of PC, and PC had no clinical significance, however, this study exhibited an important limitation: they only selected benign prostatic hyperplasia patients with severe symptoms requiring surgical treatment (23). Similarly, another study showed that the presence of PC was not a predictor of moderate/severe LUTS, but the increased calculi burden, which the researchers defined as the sum of the transverse diameters of all visible calculi within the prostate, might be associated with aggravating urinary storage symptoms, a possible explanation for this is that PC may cause prostatic inflammation (25). As can be seen, there are a variety views concerning the relationship between PC and LUTS. The inflammatory response, the size and location of PC, and prostate blood flow should be considered. The key disputes are listed in Table 1.

Chronic prostatitis (CP) is one of the most common diseases in men, with an incidence of 2.2-9.7\% (35). Chronic pelvic pain syndrome (CPPS) is among the most common types of prostatitis, and often occurs with forms of LUTS, although the pathogenesis is presently unknown (36), many factors were considered to be involved. The presence of PC is closely related to CP/CPPS, and PC associated with chronic prostate inflammation may be accompanied by chronic pelvic pain. Some studies reported that PC can frequently be found in patients with CPPS $(26,34,37)$. Soric et al. reported that the IPSS questionnaire and National Institutes of Health Chronic Prostatitis Symptom Index (NIH-CPSI) questionnaire scores were higher in the group with PC compared to the group without PC, and significantly lower maximum urinary flow occurred in the group with PC (34). Fei et al. proposed that PC plays a key role in CPPS. They suggested that PC may lead to local prostate tissue injury and inflammation, and that PC could itself be the source of infection that harbors microorganisms (37). Notably, inflammation of prostate tissue and drug resistance caused by PC may also lead to the occurrence of LUTS (31,38-40). Shoskes et al. suggested that PC could contribute to the obstruction of intraprostatic ducts and the production of bacterial biofilm, and ultimately lead to chronic inflammation of prostate (38). Moreover, Han et al. explained that inflammation of the periurethral area could maximize induction of LUTS due to inflammation-induced periurethral fibrosis, and that calcification of the urethra can also give rise to urethral stiffness $(31,41,42)$. According to these points, PC can not only generate LUTS but also prolong the duration of bothersome symptoms (32).

Large or marked PC is a significant risk factor for severe LUTS $(16,26,28,30,43)$. Sun et al. enrolled 79 prostatitis patients over 50 years of age, and the composition of PC in patients with LUTS was detected by quantitative realtime polymerase chain reaction, Western blotting, and immunofluorescence. Their conclusion suggested that large PC were related to LUTS, and that calcium oxalate (the main component of PC) leads to the occurrence of large PC (16). The results of Geramoutsos et al. are consistent with those of Sun et al; that is, the small PC observed in elderly men were normal, but large PC were associated with chronic prostate inflammation and LUTS (26). Other studies have also reported this phenomenon $(10,44)$.

PC can be distributed throughout the entire prostate gland, but are more frequently observed in the transition zone than in other zones (45). Han et al. divided the location of peri-urethral calcification (PUC) into three areas (proximal, mid, and distal), and found that the mid-PUC group had worse urinary symptoms than the control group, implying that the mid-PUC area could be a potential risk factor of LUTS (33). Cha et al. also indicated that PC in the periurethral prostate transit zone may aggravate LUTS (27). For this reason, the urethra tends to straighten to reduce its resistance by reducing the prostatic urethral angle during the voiding phase. If the prostatic urethral angle remains at a fixed tone due to limited urethral movement induced by mid-periurethral calcification fibrosis by PC, LUTS may result (46-48).

Moreover, PC may change prostatic blood flow, and alteration of prostatic blood flow or vascular resistance has an impact on LUTS $(34,49)$. Wu et al. performed a prospective analysis involving 133 elderly male patients with clinically diagnosed benign prostatic hyperplasia. They concluded that the alteration of prostatic blood flow was related to the occurrence of LUTS (49). In theory, LUTS caused by hypoxia or congestion at the bottom of the bladder may be characterized by alteration of the blood flow of bilateral neurovascular bundles, and chronic hypoxicischemic changes may increase the contraction of prostate 


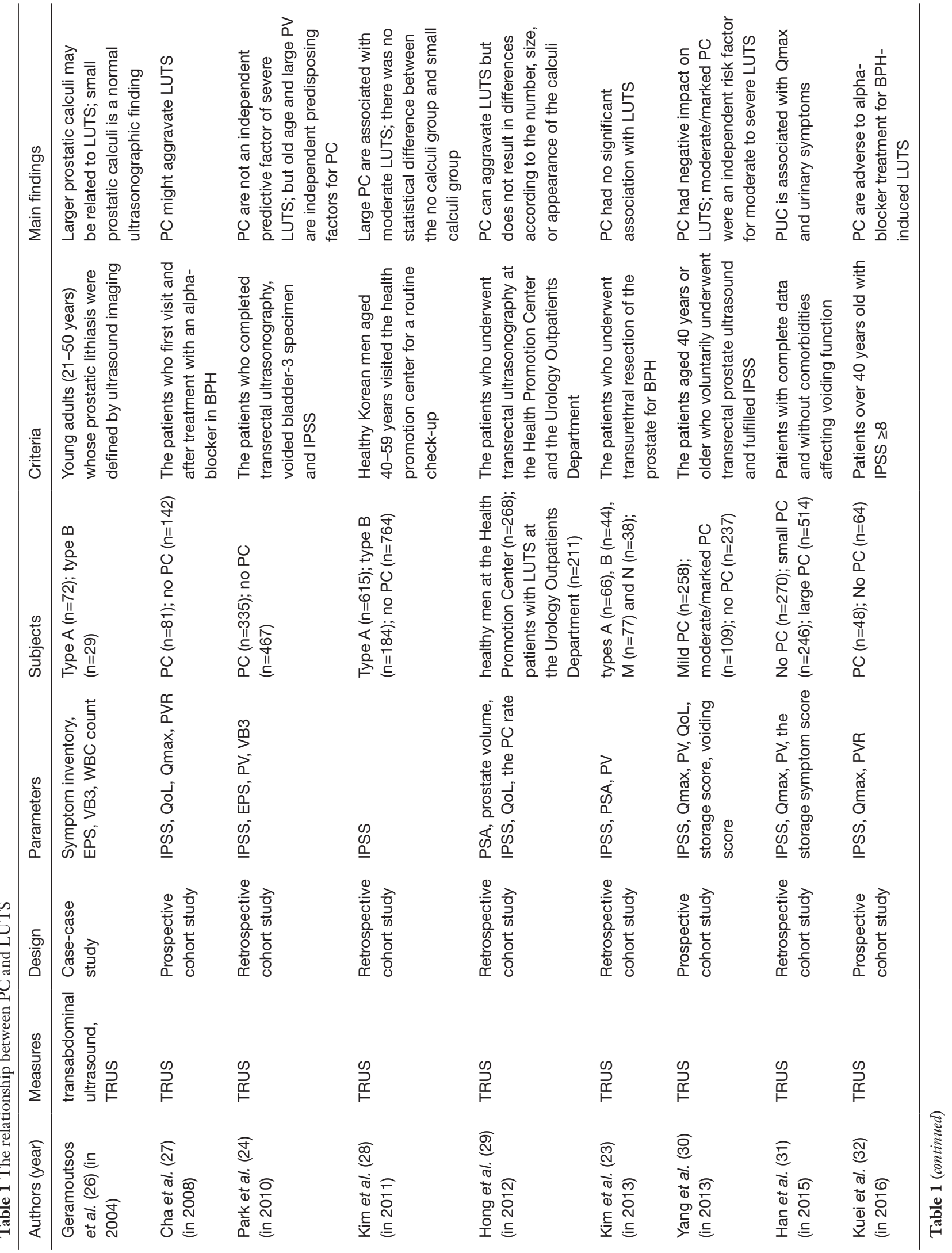




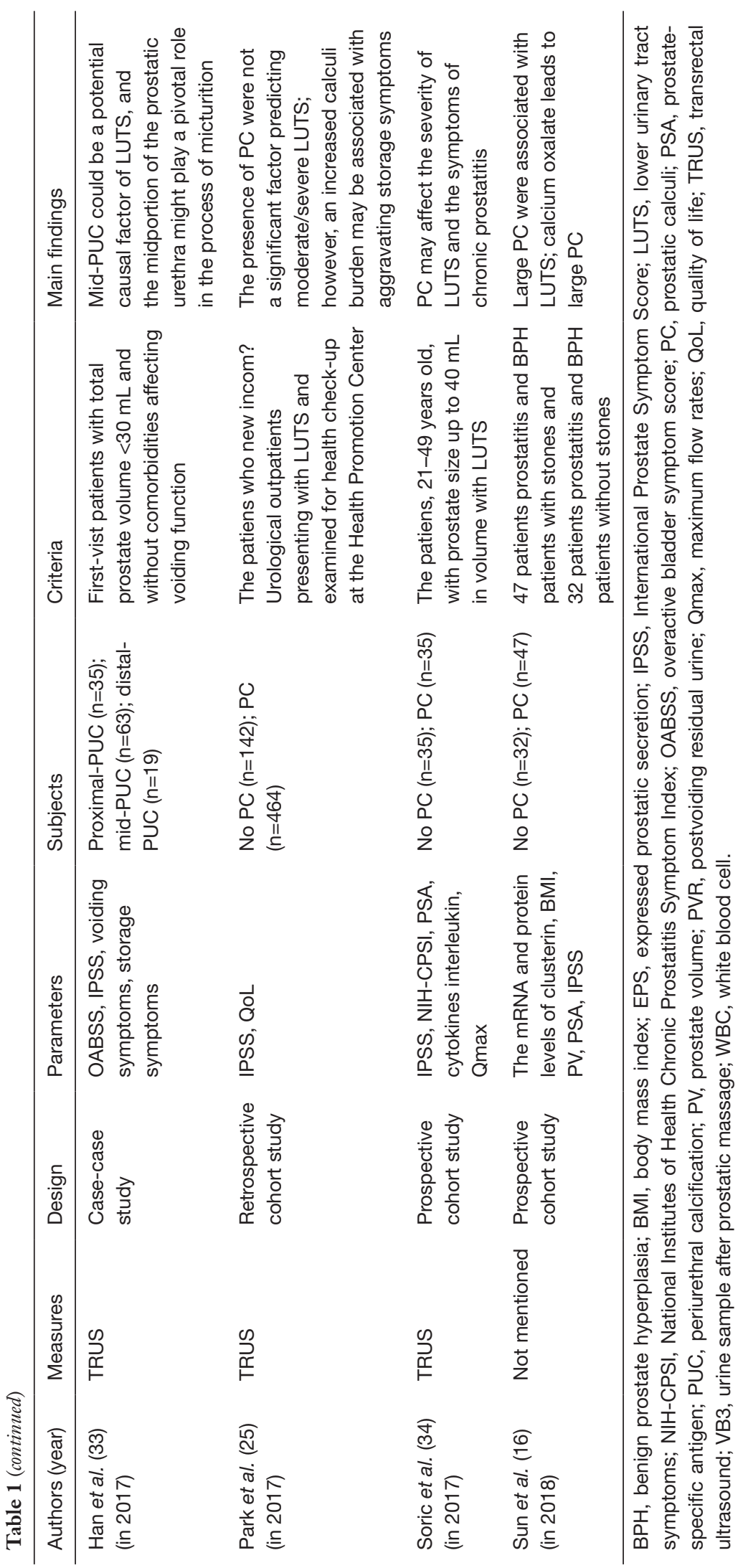




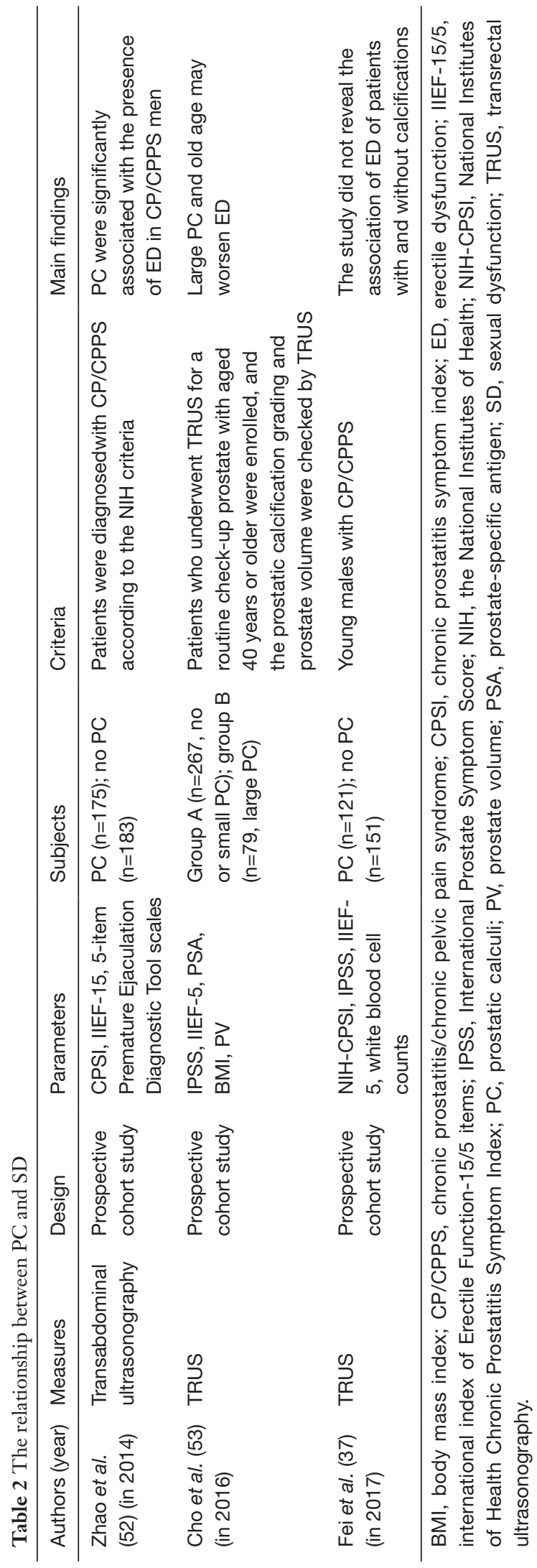

smooth muscle, both of which can cause LUTS $(50,51)$.

In summary, most studies agree that PC may affect LUTS. However, due to the lack of criteria for the definition and classification of PC, the above studies failed to uniformly define and classify PC, and thus, selection bias might have affected their results.

\section{PC and SD}

$\mathrm{SD}$ is an important component of the clinical phenotype in patients with PC. Studies exploring relationship between PC and SD are scarce, but a diverse set of related viewpoints have emerged (as shown in Table 2). PC might play a vital role in the decline of sexual function, even influence semen quality (54). Cho et al. found that middleaged men with large PC appeared to be more likely to develop ED (53). Zhao et al. discovered a significant relationship between $\mathrm{PC}$ and $\mathrm{ED}$ in a prospective study of 358 patients with CP/CPPS. They observed higher white blood cell counts or positive bacteria cultures in their prostatic fluid, longer symptom durations, and lower total scores of the International Index of Erectile Function (IIEF)-15 $(\mathrm{P}<0.001)$ were demonstrated in patients with PC. Furthermore, logistic regression analyses revealed that PC was significantly associated with self-assessed ED (52). As is often assumed, PC could cause chronic inflammation, which might affect ED by influencing smooth muscle relaxation and prostatic micro-vascularization, impairing cytokines, and enzymes, and impacting the surrounding neurovascular bundle, thus making it difficult for penile tissue to engorge and maintain erection (55-57). Another result published by Fei et al. showed that patients without PC, whose sexual function was assessed by the IIEF-5 questionnaire, responded better to medication, although they failed to verify the relationship between PC and ED (37). However, Qian et al. offered different perspectives. They proposed that psychological factors might play a key role in the pathogenesis of ED in CP/CPPS patients, and the psychological burden could occur in patients with prostatic calcification. In addition, the frequency of sexual activity may decline due to increasing depression, leading to decreased excretion of prostatic secretions (22). Neural factors should also be mentioned; an association between the presence of multiple stones of the prostatic urethra and ejaculation was reported in a case of spinal dysraphism (58). Generally, PC may have a certain effect on sexual function, but due to the lack of relevant research, the relationship between PC and sexual function is uncertain. 


\section{Discussion}

As mentioned above, $\mathrm{PC}$ have been found in many patients with LUTS or SD. The formation of PC is unclear and multifactorial. While some studies assert that $\mathrm{PC}$ are not significantly associated with LUTS or SD, the majority of studies indicate that the inflammatory response, the size and location of PC, and the blood flow of the prostate are likely the key factors causing LUTS and SD. Owing to the chronic irritation to the surrounding tissue in PC, chronic inflammation-induced prostate fibrosis aggravates LUTS and promotes the formation of new stones. Large or marked PC are found to be significantly correlated with LUTS, and the fixation of prostatic urethral angle due to PC-induced mid-periurethral calcification fibrosis is shown to be involved in LUTS. In addition, hypoxia is discovered to possibly increase prostatic smooth muscle contraction, ultimately resulting in LUTS. As for SD, chronic inflammation may affect smooth muscle relaxation, prostatic micro-vascularization, cytokines, enzymes, and blood flow of the penis, thereby leading to ED. Importantly, psychological factors cannot be ignored. Patients may experience decreased libido due to worrying about PC, which may lead to the occurrence of ED and further accelerate the formation of PC.

However, the above studies exhibit the following limitations. Firstly, due to the variety of classifications and definitions of PC, they do not define and classify PC consistently. Secondly, the different inclusion criteria used might have led to differences in the samples studied; for example, if the selected samples are elderly patients who require surgery due to benign prostatic hyperplasia, patients with mild LUTS or asymptomatic patients may be ignored. Thirdly, some studies were retrospective studies and contained a small sample size, and thus methodological deficiencies of included studies should be considered as a major limitation of this review. In addition, most of the articles were based on clinical data, which might have led to selection and information biases, with the study's conclusion also lacking the support of basic research. Thus, more indepth studies of the molecular mechanisms are needed.

For future research, the following aspects require further attention. (I) The development of consistent criteria for defining and classifying PC is crucial. This includes the same instruments being used to measure and obtain a unified diameter of each category. (II) Multicenter, largesample, prospective, controlled, and longitudinal studies should be carried out. For instance, prospective cohort studies should be conducted to determine whether PC are risk factors for LUTS, and intervention studies that remove PC of patients with LUTS should be performed to validate whether this is effective. (III) The relationship between PC and LUTS/SD should be examined using pathological studies to evaluate the clinical significance and explore the molecular mechanisms. Finally, (IV) the influence of psychological factors, such as anxiety disorder caused by $\mathrm{PC}$, requires further elucidation by experiments.

\section{Conclusions}

Through a literature review, the relationship between PC and LUTS/SD was summarized. The results in this review indicate that most studies agree that PC may be a risk factor for LUTS and SD. We also expounded the relevant mechanisms by reviewing the available literature, and determined that PC may aggravate LUTS and SD. The size and location of PC, inflammation, changes in prostatic blood flow, and psychological factors should be fully considered, and action could be taken to treat LUTS and $\mathrm{SD}$ in order to reduce the incidence of PC.

\section{Acknowledgments}

Funding: This work was supported by the Natural Science Foundation of China (81871147 \& 81671453), Sichuan Science and Technology Program (2018TJPT0018), and Chengdu Science and Technology Program (2019-YFYF00087-SN).

\section{Footnote}

Reporting Checklist: The authors have completed the NARRATIVE REVIEW reporting checklist. Available at http://dx.doi.org/10.21037/tau-20-1046

Conflicts of Interest: All authors have completed the ICMJE uniform disclosure form (available at http://dx.doi. org/10.21037/tau-20-1046). The authors have no conflicts of interest to declare.

Ethical Statement: The authors are accountable for all aspects of the work in ensuring that questions related to the accuracy or integrity of any part of the work are appropriately investigated and resolved.

Open Access Statement: This is an Open Access article 
distributed in accordance with the Creative Commons Attribution-NonCommercial-NoDerivs 4.0 International License (CC BY-NC-ND 4.0), which permits the noncommercial replication and distribution of the article with the strict proviso that no changes or edits are made and the original work is properly cited (including links to both the formal publication through the relevant DOI and the license). See: https://creativecommons.org/licenses/by-nc-nd/4.0/.

\section{References}

1. Klimas R, Bennett B, Gardner WA, Jr. Prostatic calculi: a review. Prostate 1985;7:91-6.

2. Stamatiou K, Magri V, Perletti G, et al. Prostatic calcifications are associated with a more severe symptom burden in men with type II chronic bacterial prostatitis. Arch Ital Urol Androl 2019. doi: 10.4081/aiua.2019.2.79.

3. Hyun JS. Clinical Significance of Prostatic Calculi: A Review. World J Mens Health 2018;36:15-21.

4. O'Neill AGM, Osman SO, Jain S, et al. Observed high incidence of prostatic calculi with the potential to act as natural fiducials for prostate image guided radiotherapy. Tech Innov Patient Support Radiat Oncol 2019;9:35-40.

5. Tang Z, Wu XM, Wei Q, et al. The prevalence and risk factors of prostatic calcification: an analysis of 68705 subjects. Asian J Androl 2018;20:417-9.

6. Meng J, Zhang M, Guan SY, et al. The prevalence and risk factors of prostatic calculi in Han Chinese: a crosssectional study based on health examinations. Aging Male 2019. [Epub ahead of print].

7. Vilches J, Lopez A, De Palacio L, et al. SEM and $\mathrm{X}$-ray microanalysis of human prostatic calculi. J Urol 1982;127:371-3.

8. Harada K, Igari D, Tanahashi Y. Gray scale transrectal ultransonography of the prostate. J Clin Ultrasound 1979;7:45-9.

9. Torres Ramirez C, Aguilar Ruiz J, Zuluaga Gomez A, et al. A crystallographic study of prostatic calculi. J Urol 1980;124:840-3.

10. Najoui M, Qarro A, Ammani A, et al. Giant prostatic calculi. Pan Afr Med J 2013;14:69.

11. Chute CG, Panser LA, Girman CJ, et al. The prevalence of prostatism: a population-based survey of urinary symptoms. J Urol 1993;150:85-9.

12. Egan KB. The Epidemiology of Benign Prostatic Hyperplasia Associated with Lower Urinary Tract Symptoms: Prevalence and Incident Rates. Urol Clin North Am 2016;43:289-97.
13. Kirk D, McNicholas TA. Benign prostatic hyperplasia: counting the cost of its management. BJU Int 2010;106:586.

14. McCabe MP, Sharlip ID, Lewis R, et al. Incidence and Prevalence of Sexual Dysfunction in Women and Men: A Consensus Statement from the Fourth International Consultation on Sexual Medicine 2015. J Sex Med 2016;13:144-52.

15. McCabe MP, Sharlip ID, Atalla E, et al. Definitions of Sexual Dysfunctions in Women and Men: A Consensus Statement From the Fourth International Consultation on Sexual Medicine 2015. J Sex Med 2016;13:135-43.

16. Sun C, Xie G, Huang F, et al. Effects of Calcium Oxalate on Expression of Clusterin and Lower Urinary Tract Symptoms in Prostatitis and Benign Prostatic Hyperplasia Patients with Calculi. Med Sci Monit 2018;24:9196-203.

17. Koseoglu H, Aslan G, Sen BH, et al. [Prostatic calculi: silent stones]. Actas Urol Esp 2010;34:555-9.

18. Balasar M, Sonmez MG, Aydin A, et al. Is There A Relation Between Serum Uric Acid Values and Prostatic Calculi Presence? Urol Int 2019;102:199-204.

19. Akbaba AI, Ozgul RK, Dursun A. Presentation of 14 alkaptonuria patients from Turkey. J Pediatr Endocrinol Metab 2020;33:289-94.

20. Sridhar FK, Mukha RP, Kumar S, et al. Lower urinary tract symptoms and prostatic calculi: A rare presentation of alkaptonuria. Indian J Urol 2012;28:219-21.

21. Cao JJ, Huang W, Wu HS, et al. Prostatic Calculi: Do They Matter? Sex Med Rev 2018;6:482-91.

22. Qian S, Tang Z, Yuan J. Re: A prospective study on association of prostatic calcifications with sexual dysfunction in men with chronic prostatitis/chronic pelvic pain syndrome (CP/CPPS). J Sex Med 2014;11:3127.

23. Kim SH, Jung KI, Koh JS, et al. Lower urinary tract symptoms in benign prostatic hyperplasia patients: orchestrated by chronic prostatic inflammation and prostatic calculi? Urol Int 2013;90:144-9.

24. Park SW, Nam JK, Lee SD, et al. Are prostatic calculi independent predictive factors of lower urinary tract symptoms? Asian J Androl 2010;12:221-6.

25. Park B, Choo SH. The burden of prostatic calculi is more important than the presence. Asian J Androl 2017;19:482-5.

26. Geramoutsos I, Gyftopoulos K, Perimenis P, et al. Clinical correlation of prostatic lithiasis with chronic pelvic pain syndromes in young adults. Eur Urol 2004;45:333-7; discussion 337-8.

27. Cha WH, Kim KH, Seo YJ. The effect of periurethral 
prostatic calculi on lower urinary tract symptoms in benign prostatic hyperplasia. Korean J Urol 2008;49:237-41.

28. Kim WB, Doo SW, Yang WJ, et al. Influence of prostatic calculi on lower urinary tract symptoms in middle-aged men. Urology 2011;78:447-9.

29. Hong CG, Yoon BI, Choe HS, et al. The Prevalence and Characteristic Differences in Prostatic Calcification between Health Promotion Center and Urology Department Outpatients. Korean J Urol 2012;53:330-4.

30. Yang HJ, Huang KH, Wang CW, et al. Prostate calcification worsen lower urinary tract symptoms in middle-aged men. Urology 2013;81:1320-4.

31. Han JH, Kwon JK, Lee JY, et al. Is periurethral calcification associated with urinary flow rate and symptom severity in men with lower urinary tract symptoms-benign prostatic hyperplasia? A retrospective review. Urology 2015;85:1156-61.

32. Kuei $\mathrm{CH}$, Liao CH, Chiang BJ. Significant intravesical prostatic protrusion and prostatic calcification predict unfavorable outcomes of medical treatment for male lower urinary tract symptoms. Urol Sci 2016;27:13-6.

33. Han JH, Lee JY, Kwon JK, et al. Clinical Significance of Periurethral Calcification According to the Location in Men With Lower Urinary Tract Symptoms and a Small Prostate Volume. Int Neurourol J 2017;21:220-8.

34. Soric T, Selimovic M, Bakovic L, et al. Clinical and Biochemical Influence of Prostatic Stones. Urol Int 2017;98:449-55.

35. Krieger JN, Lee SWH, Jeon J, et al. Epidemiology of prostatitis. Int J Antimicrob Agents 2008;31 Suppl 1:S85-90.

36. Bartoletti R, Cai T, Mondaini N, et al. Prevalence, incidence estimation, risk factors and characterization of chronic prostatitis/chronic pelvic pain syndrome in urological hospital outpatients in Italy: results of a multicenter case-control observational study. J Urol 2007;178:2411-5; discussion 2415.

37. Fei $X$, Jin W, Hua S, et al. Prospective Study on Association of Prostatic Calcifications with Clinical Symptoms and Results of Treatment in Men with type III prostatitis. Sci Rep 2017;7:5234.

38. Shoskes DA, Lee CT, Murphy D, et al. Incidence and significance of prostatic stones in men with chronic prostatitis/chronic pelvic pain syndrome. Urology 2007;70:235-8.

39. Donnell RF. Antinanobacterial therapy for men with chronic prostatitis/chronic pelvic pain syndrome and prostatic stones. Curr Urol Rep 2005;6:279-80.
40. Schaeffer AJ, Landis JR, Knauss JS, et al. Demographic and clinical characteristics of men with chronic prostatitis: the national institutes of health chronic prostatitis cohort study. J Urol 2002;168:593-8.

41. Forman JL, Kent RW. The effect of calcification on the structural mechanics of the costal cartilage. Comput Methods Biomech Biomed Engin 2014;17:94-107.

42. Sekikawa A, Shin C, Curb JD, et al. Aortic stiffness and calcification in men in a population-based international study. Atherosclerosis 2012;222:473-7.

43. Den J, Kerr PS, Dafashy TJ, et al. Urethral Steinstrasse following Laser Lithotripsy of Prostatic Urethral Calculi. Case Rep Urol 2018;2018:3459347.

44. Malle M, Fofana Y, Diallo M, et al. Voluminous juvenile prostatic lithiasis complicated by perineal fistula: about a case reported at the regional hospital in Gao. Pan Afr Med J 2019;32:23.

45. Suh JH, Gardner JM, Kee KH, et al. Calcifications in prostate and ejaculatory system: a study on 298 consecutive whole mount sections of prostate from radical prostatectomy or cystoprostatectomy specimens. Ann Diagn Pathol 2008;12:165-70.

46. Ukimura O, Iwata T, Ushijima S, et al. Possible contribution of prostatic anterior fibromuscular stroma to age-related urinary disturbance in reference to pressureflow study. Ultrasound Med Biol 2004;30:575-81.

47. Cho KS, Kim JH, Kim DJ, et al. Relationship between prostatic urethral angle and urinary flow rate: its implication in benign prostatic hyperplasia pathogenesis. Urology 2008;71:858-62.

48. Kang DH, Lee JY, Hah YS, et al. Correlation of prostatic urethral angle with the severity of urinary symptom and peak flow rate in men with small prostate volume. PLoS One 2014;9:e104395.

49. Wu KY, Tsai YS, Chen CH, et al. Association of Prostate Blood Flow with Male Lower Urinary Tract Symptoms. Urol Int 2016;97:352-7.

50. Azadzoi KM, Babayan RK, Kozlowski R, et al. Chronic ischemia increases prostatic smooth muscle contraction in the rabbit. J Urol 2003;170:659-63.

51. Neumaier CE, Martinoli C, Derchi LE, et al. Normal prostate gland: examination with color Doppler US. Radiology 1995;196:453-7.

52. Zhao Z, Xuan X, Zhang J, et al. A prospective study on association of prostatic calcifications with sexual dysfunction in men with chronic prostatitis/chronic pelvic pain syndrome (CP/CPPS). J Sex Med 2014;11:2528-36.

53. Cho YH, Sohn DW, Kim SW. Large prostatic calculi 
may worsen erectile dysfunction and lower urinary tract symptoms in middle aged men. Int J Urol 2016;23:4-5.

54. Chen T, Tian L, Bai G, et al. Clinical Correlation of Prostatic Calculi With Semen Parameters in Adult Men With Fertility Intention. Am J Mens Health 2019;13:1557988319852018.

55. Chung SD, Keller JJ, Lin HC. A case-control study on the association between chronic prostatitis/chronic pelvic pain syndrome and erectile dysfunction. BJU Int 2012;110:726-30.

56. Sprague AH, Khalil RA. Inflammatory cytokines in

Cite this article as: Wang H, Ma M, Qin F, Yuan J. The influence of prostatic calculi on lower urinary tract symptoms and sexual dysfunction: a narrative review. Transl Androl Urol 2021;10(2):929-938. doi: 10.21037/tau-20-1046 vascular dysfunction and vascular disease. Biochem Pharmacol 2009;78:539-52.

57. Tran CN, Shoskes DA. Sexual dysfunction in chronic prostatitis/chronic pelvic pain syndrome. World J Urol 2013;31:741-6.

58. Ahsaini M, Mellouki A, Mellas S, et al. Multiple stones of the prostatic urethra associated with anejaculation revealing spinal dysraphism such as tethered cord syndrome at the base of the spinal canal in a young man: about an exceptional case. Pan Afr Med J 2019;33:165. 